\title{
Medium polarization effects on neutron matter superfluidity
}

\author{
H.-J. Schulze ${ }^{a}$, J. Cugnon ${ }^{a}$, A. Lejeune ${ }^{a}$, M. Baldo ${ }^{b}$, U. Lombardo ${ }^{b}$ \\ a Université de Liège, Institut de Physique B5, Sart Tilman, B-4000 Liège 1, Belgium

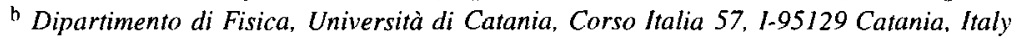 \\ Received 4 January 1996; revised manuscript received 12 February 1996 \\ Editor: W. Haxton
}

\begin{abstract}
We solve the ${ }^{1} S_{0}$ gap equation for neutron matter using an effective interaction based on the Argonne $V_{14}$ potential that includes effects of the polarization (RPA) graphs. We find a substantial reduction of the gap, and a slight extension of the domain of superfluidity to larger densities. We demonstrate the inadequacy of using the weak coupling approximation.
\end{abstract}

PACS: $21.60 . \mathrm{Jz}: 21.90 .+\mathrm{f}$

Keywords: Neutron matter; Superfluidity; Polarization effects

The superfluid properties of neutron matter are an important issue for the understanding of neutron star physics [1]. This problem is usually studied in the BCS theory [2] or using the Green functions techniques [3-5]. Both yield the same gap equation for the modified quasi-particle spectrum, but the latter has the advantage of providing a more or less systematic way of building the pairing interaction in a series of the bare interaction. The first term is the bare potential itself $[4,6]$. At this level, some agreement exists between the various results in the literature, provided the gap equation is solved exactly and a realistic nucleonnucleon interaction is used [7-9].

The necessity of going beyond this approximation is rather clear, as the next approximations involve the polarisability (with respect to density and spin-density fluctuations), which is expected, as for nuclear matter, to play an important role. Yet there was initially no clear guideline to select the most important higher order graphs, until the Babu-Brown theory [10] for the effective interaction appeared. These authors showed that the exchange of the RPA particle-hole series between two particles is not a good description of the effective interaction, as it produces a too strong polarizability, if not an instability, of nuclear matter. They proposed to include into the particle-hole channel, the so-called induced interaction, which guarantees antisymmetry and Landau sum rules, and which makes the nuclear matter Landau parameter $F_{0}$ less negative.

As for the ${ }^{1} S_{0}$ pairing in neutron matter, the first calculations along these lines were rather confusing, as they predicted an enhancement $[11,12]$ or a reduction [13] of the pairing interaction. These works are however based on simple estimates of the Landau parameters. A more detailed approach, performed by Chen et al. [7], including only the second order graphs, but in a correlated basis formalism (and without resorting on the Landau parameters) concluded to a strong reduction of the ${ }^{1} S_{0}$ gap. Later. Ainsworth et al. [14] performed an almost complete study of the effective interaction, not restricting to the Fermi surface. They however finally used the so-called weak-coupling for- 


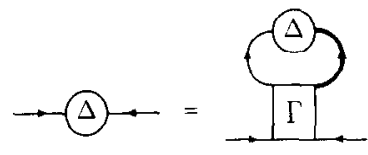

(a)

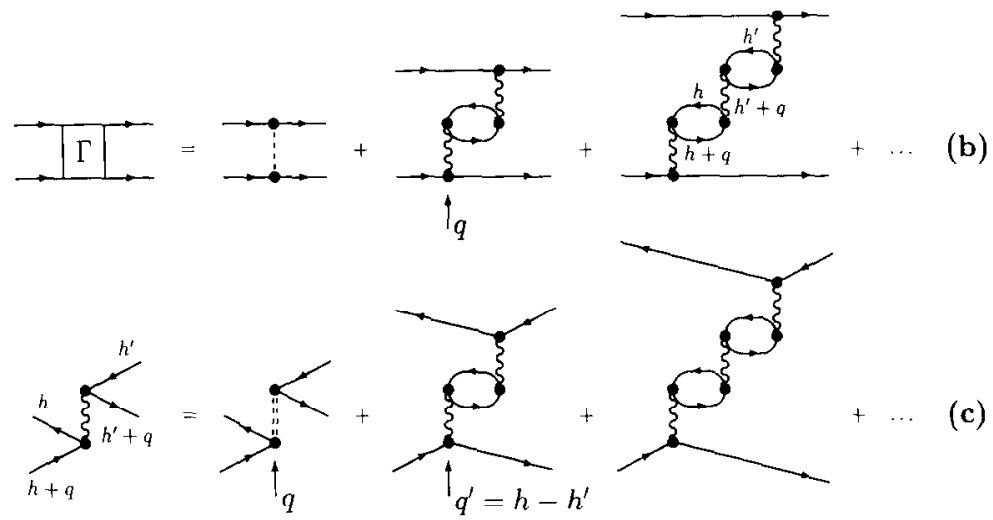

Fig. 1. A graphical representation of our approximation scheme: (a): The gap equation in the Green function formalism. The irreducible interaction kernel $\Gamma$ is further specified in (b): The lowest order graph (dashed line) is the bare potential in the ${ }^{1} S_{0}$ channel, whereas the particle-hole interaction in the polarization diagrams (wiggly line) is given by the Babu-Brown like equation shown in (c): Here the double-dashed line represents phase-space averaged, antisymmetrized $G$-matrix elements.

mula to estimate the gap. They concluded on a sizable reduction also.

The purpose of this article is to provide a new calculation mostly along the lines of Ref. [14], i.e., we will construct an effective, exchanged-momentum dependent interaction that includes in an approximate way the effect of the polarization diagrams shown in Fig. 1. However, this interaction will be used without further approximation in the kernel of the full gap equation. We will demonstrate the importance of retaining the full momentum dependence of the effective interaction, in contrast to restricting it to the Fermi surface, as using the Landau parameters, to estimate the change of the gap. We also demonstrate the complete failure of the weak-coupling approximation in our model. It is already known from the solution of the gap equation for the lowest order, "direct", interaction, that this formula may yield quantitatively wrong results $[8]$, unless special account of the off-diagonal part of the interaction is made.

Our aim is the solution of the gap equation (shown graphically in Fig. 1(a)) in the ${ }^{1} S_{0}$ channel,

$$
\begin{aligned}
& \Delta(k)=-\frac{1}{4 \pi^{2}} \int_{0}^{\infty} d k^{\prime} k^{\prime 2} \Gamma_{1_{S_{0}}}\left(k, k^{\prime}\right) \\
& \times \frac{\Delta\left(k^{\prime}\right)}{\sqrt{\left[e\left(k^{\prime}\right)-e\left(k_{0}\right)\right]^{2}+\Delta\left(k^{\prime}\right)^{2}}}, \\
& \rho=\frac{k_{F}^{3}}{3 \pi^{2}}=\frac{1}{\pi^{2}} \int_{0}^{\infty} d k k^{2} \\
& \quad \times \frac{1}{2}\left[1-\frac{\left[e(k)-e\left(k_{0}\right)\right]}{\sqrt{\left[e(k)-e\left(k_{0}\right)\right]^{2}+\Delta(k)^{2}}}\right],
\end{aligned}
$$

i.e., the determination of the gap function $\Delta(k)$ and the chemical potential $\mu=e\left(k_{0}\right)$ for a given neutron density $\rho$ or neutron Fermi momentum $k_{F}$. Here $e(k)$ is the neutron single-particle energy, provided by an independent Brueckner-Hartree-Fock (BHF) $G$-matrix calculation. The same $G$-matrix elements will be used in the following for the construction of the polarization part of the effective interaction used in the gap equation.

For the simple case of a central potential acting in spin symmetric and antisymmetric channels,

$$
V=V_{s}(r)+V_{a}(r) \boldsymbol{\sigma}_{1} \cdot \boldsymbol{\sigma}_{2} .
$$


the interaction in the ${ }^{\mathrm{l}} S_{0}$ channel is equal, in lowest order (first graph of the series of Fig. 1(b)), to the isotropic part of a partial wave expansion

$\Gamma_{\mathcal{S}_{i \mid}}\left(k, k^{\prime}\right)=\frac{1}{2 k k^{\prime}} \int_{\left|k-k^{\prime}\right|}^{k+k^{\prime}} d q q\left[\widetilde{V}_{s}(q)-3 \widetilde{V}_{a}(q)\right]$,

with the Fourier transforms $(i=s, a)$

$\widetilde{V}_{i}(q)=4 \pi \int_{0}^{\infty} d r r^{2} j_{0}(q r) V_{i}(r)$

The solution of the gap equation with this lowest order,"direct", interaction has been given in a number of publications for various realistic nucleon-nucleon interactions $[7,8,15]$. One obtains typically a maximum of the gap $\Delta_{F}=\Delta\left(k_{F}\right) \approx 3 \mathrm{MeV}$ at a density corresponding to $k_{F} \approx 0.85 \mathrm{fm}^{-1}$. However, the use of the bare potential completely disregards the influence of the polarizability of the neutron medium. To account for this, we closely follow the approach of refs $[16,17]$, constructing the pairing interaction $\Gamma$ in three steps:

(i) We first construct a local approximation of the neutron-neutron G-matrix. The practical importance of having a local effective interaction will become clear in a moment. Let us consider first the $G$-matrix elements for a given spin $S$ channel:

$$
\begin{aligned}
& \frac{1}{2 S+1} \sum_{m_{S}}\left\langle S, m_{S}, k^{\prime}|G(p, W)| k, S, m_{S}\right\rangle_{A} \\
& =\frac{2}{2 S+1} \sum_{J=0}^{\infty} \sum_{L=J, J \pm 1}(2 J+1) P_{L}(z) \\
& \times G_{S, L, J}\left(W, p, k, k^{\prime}\right) .
\end{aligned}
$$

There is a dependence on the total momentum $p$, the total (starting) energy $W$, the relative momenta of initial and final state $k$ and $k^{\prime}$, and the scattering angle $z=\cos \theta=\hat{\boldsymbol{k}} \hat{\boldsymbol{k}}^{\prime}$. Besides, the matrix elements depend on the discrete quantum numbers $S(=0,1), L$ (even (odd) for $S=0(1)$ ), and $J$. The factor 2 on the rhs is due to antisymmetrization, and we have not indicated the complications due to the tensor interaction. The matrix elements $G_{S, L, J}\left(p, W, k, k^{\prime}\right)$ are obtained by numerically solving the BHF scheme for partial waves up to ${ }^{3} \mathrm{H}_{4}$.

Thus, in order to be able to use the simple formula Eq. (16), we have to devise an averaging procedure in order to construct an approximate effective local interaction of the form Eq. (2) from the complicated $G$-matrix elements. This procedure will be based on simple phase-space averaging of the kinematical arguments of the $G$-matrix. As shown in Fig. I (b), we average the $G$-matrix that converts a particle-hole pair with momenta $\boldsymbol{h}+\boldsymbol{q}, \boldsymbol{h}$ into one with momenta $\boldsymbol{h}^{\prime}+\boldsymbol{q}$, $\boldsymbol{h}^{\prime}$. The relative and total momenta, and the starting energy are in this case:

$$
\begin{aligned}
& \boldsymbol{k}^{\prime}=\boldsymbol{k}+\boldsymbol{q}=\frac{1}{2}\left(\boldsymbol{h}^{\prime}-\boldsymbol{h}-\boldsymbol{q}\right), \\
& \boldsymbol{p}=\boldsymbol{h}+\boldsymbol{h}^{\prime}+\boldsymbol{q}, \\
& \boldsymbol{q}^{\prime}=\boldsymbol{h}^{\prime}-\boldsymbol{h}, \\
& W=e(h)+e\left(h^{\prime}\right)
\end{aligned}
$$

(the last equation being valid since we work in the instantaneous approximation $\left.\left(q_{0}=0\right)\right)$. We have also listed here the momentum transfer in the exchange channel, $\boldsymbol{q}^{\prime}$, that will be of relevance further below.

We now define a typical value of a kinematical quantity $a\left(\boldsymbol{h}, \boldsymbol{h}^{\prime}, \boldsymbol{q}\right)$ by phase-space averaging as

$$
\begin{aligned}
& \langle a\rangle_{q}:= \\
& \frac{\int d^{3} h \frac{f(\boldsymbol{h}) \bar{f}(\boldsymbol{h}+q)}{e(\boldsymbol{h})-e(\boldsymbol{h}+\boldsymbol{q})} \int d^{3} h^{\prime} \frac{f\left(\boldsymbol{h}^{\prime}\right) \bar{f}\left(\boldsymbol{h}^{\prime}+q\right)}{e\left(\boldsymbol{h}^{\prime}\right)-e\left(\boldsymbol{h}^{\prime}+\boldsymbol{q}\right)} a\left(\boldsymbol{h}, \boldsymbol{h}^{\prime}, \boldsymbol{\varphi}\right)}{\int d^{3} h \frac{f(\boldsymbol{h}) \bar{f}(\boldsymbol{h}+q)}{e(\boldsymbol{h})-e(\boldsymbol{h}+q)} \int d^{3} h^{\prime} \frac{f\left(\boldsymbol{h}^{\prime}\right) \bar{f}\left(\boldsymbol{h}^{\prime}+q\right)}{e\left(\boldsymbol{h}^{\prime}\right)-e\left(\boldsymbol{h}^{\prime}+q\right)}}
\end{aligned}
$$

with $f(\boldsymbol{h})=\Theta\left(k_{F}-|\boldsymbol{h}|\right)$ and $\bar{f}=1-f$. The energy denominators in these expressions represent the probability of exciting a virtual particle-hole pair. Employing an effective mass approximation for the singleparticle energies, one is able to carry out analytically the six-dimensional integrations above. The results are

$$
\begin{aligned}
& \left\langle p^{2}\right\rangle_{q}=2\left\langle h^{2}\right\rangle_{q}+2\langle h z\rangle_{q}^{2}+4 q\langle h z\rangle_{q}+q^{2}, \\
& \left\langle k^{2}\right\rangle_{q}=\left\langle k^{2}\right\rangle_{q}=\frac{1}{4}\left[2\left\langle h^{2}\right\rangle_{q}-2\langle h z\rangle_{q}^{2}+q^{2}\right], \\
& \left\langle\boldsymbol{k} \boldsymbol{k}^{\prime}\right\rangle_{q}=\frac{1}{4}\left[2\left\langle h^{2}\right\rangle_{q}-2\langle h z\rangle_{q}^{2}-q^{2}\right], \\
& \left\langle q^{\prime 2}\right\rangle_{q}=2\left\langle h^{2}\right\rangle_{q}-2\langle h z\rangle_{q}^{2}, \\
& \langle z\rangle_{q}:=\frac{\left\langle\boldsymbol{k} \boldsymbol{k}^{\prime}\right\rangle_{q}}{\left\langle k^{2}\right\rangle_{q}},
\end{aligned}
$$


$\langle W\rangle_{q}:=2 e\left(\langle h\rangle_{q}\right), \quad\langle h\rangle_{q}:=\sqrt{\left\langle h^{2}\right\rangle_{q}}$.

with

$$
\begin{aligned}
\left\langle h^{2}\right\rangle_{4} & =\frac{\Pi^{\left(h^{2}\right)}(q)}{\Pi(q)}, \\
\langle h z\rangle_{4} & =\frac{\Pi^{(h z)}(q)}{\Pi(q)}
\end{aligned}
$$

and (with $x=q / 2 k_{F}$ )

$$
\begin{aligned}
& \Pi(q)=N \pi(x), \quad N=-\frac{m^{*} k_{F}}{\pi^{2}}, \\
& \pi(x)=\frac{1}{2}\left[1+\frac{1-x^{2}}{2 x} \log \left|\frac{1+x}{1-x}\right|\right], \\
& \Pi^{(h-1}(q)=\left\{\begin{array}{c}
k_{F} N\left[\frac{1}{2}-\frac{x^{2}}{6}-x \pi(x)\right], \\
x<1 \\
k_{F} N\left[\frac{1}{3 x}-x \pi(x)\right], \\
x>1
\end{array}\right. \\
& \Pi^{\left(h^{2}\right)}(q)=\left\{\begin{array}{c}
k_{F}^{2} N\left[\frac{1}{2}-x+\frac{x^{3}}{3}+\frac{x^{2}+1}{2} \pi(x)\right], \\
x<1 \\
k_{F}^{2} N\left[-\frac{1}{6}+\frac{x^{2}+1}{2} \pi(x)\right], \\
x>1
\end{array}\right.
\end{aligned}
$$

In these expressions, $\Pi(q)$ is the Lindhard function at zero energy transfer, and $N$ the density of states at the Fermi surface for neutron matter. A plot of the various averaged quantities as a function of $q$ is shown in Fig. 2. We can now use these averaged quantities in order to define phase-space averaged $G$-matrix elements as well, namely by evaluating the right hand side of Eq. (5) with the averaged kinematical quantities inserted:

$$
\begin{aligned}
& \left\langle G^{S}\right\rangle_{q}:=\frac{2}{2 S+1} \sum_{J=0}^{\infty} \sum_{L=J, J \pm 1}(2 J+1) P_{L}\left(\langle z\rangle_{q}\right) \\
& \times G_{S, L, J}\left(\langle W\rangle_{q},\langle p\rangle_{q},\langle k\rangle_{q},\left\langle k^{\prime}\right\rangle_{q}\right) .
\end{aligned}
$$

The averaging procedure accordingly selects for given momentum transfer $q$ "typical" matrix elements of the interaction in the various channels. In particular it is not restricted to the surface of the Fermi sphere. This

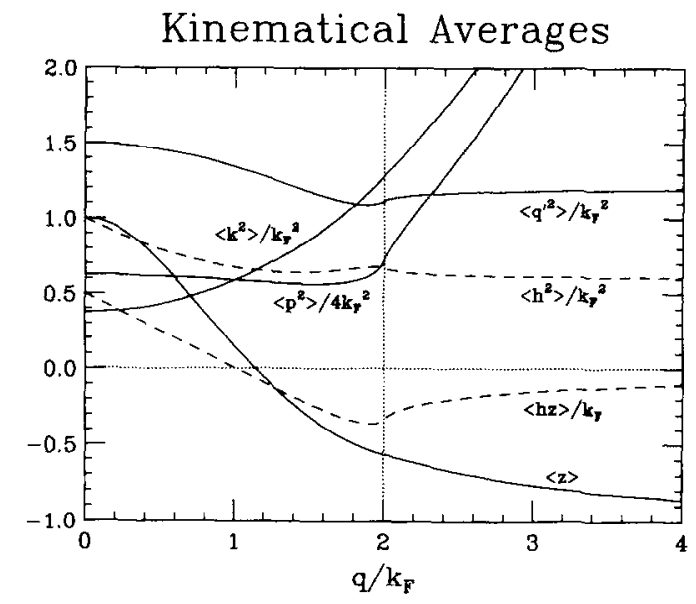

Fig. 2. Various phase-space averaged kinematical quantities as given by Eqs. (8) and (9).

is perhaps a questionable prescription, al though the $G$ matrix might be close to be local [17]. Note however that this averaging prescription is exact for the case of a local interaction, i.e., $\langle V\rangle_{q}=V(q)$, due to the definition of the averaged scattering angle Eq. (8e), which ensures that $2\left\langle k^{2}\right\rangle_{q}\left(1-\langle z\rangle_{q}\right)=q^{2}$. On the other hand it automatically includes the exchange graphs by the use of antisymmetrized $G$-matrix elements in Eq. (11).

From the averaged $G$-matrix elements in the $S=$ 0,1 channels we can finally define effective interactions in the spin-symmetric and antisymmetric channels, cf. Eq. (2), by

$$
\begin{aligned}
& G_{s}(q)=\frac{1}{4}\left[3\left\langle G^{S=1}\right\rangle_{q}+\left\langle G^{S=0}\right\rangle_{q}\right], \\
& G_{a}(q)=\frac{1}{4}\left[\left\langle G^{S=1}\right\rangle_{q}-\left\langle G^{S=0}\right\rangle_{q}\right] .
\end{aligned}
$$

(ii) We construct the effective interaction in the particle-hole channel, i.e. the series of Fig. $I(\mathrm{c})$. Very much along the same lines as in Ref. [14], the latter, for $q=0$, is given by (disregarding for a while the spin structure)

$$
\begin{aligned}
& F\left(\boldsymbol{k}_{1}, \boldsymbol{k}_{2}\right)=G\left(\boldsymbol{k}_{1}, \boldsymbol{k}_{2}\right) \\
& -\sum_{n=2}^{\infty} \sum_{\boldsymbol{K}_{1}, \ldots \boldsymbol{K}_{n-1}} F\left(\frac{\boldsymbol{k}_{1}+\boldsymbol{k}_{2}}{2}, \boldsymbol{K}_{1}\right) \\
& \quad \times \frac{f\left(\boldsymbol{K}_{1}+\boldsymbol{q}^{\prime} / 2\right)-f\left(\boldsymbol{K}_{1}-\boldsymbol{q}^{\prime} / 2\right)}{\boldsymbol{K}_{1} \cdot \boldsymbol{q}^{\prime} / 2 m^{*}} F\left(\boldsymbol{K}_{1}, \boldsymbol{K}_{2}\right) \ldots
\end{aligned}
$$




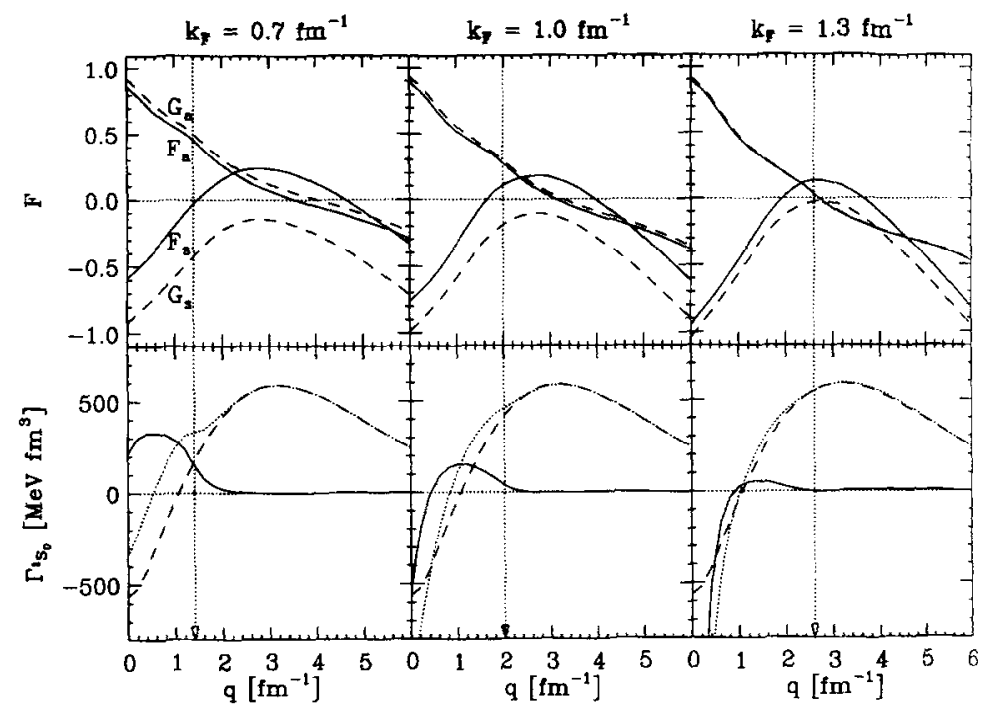

Fig. 3. The particle-hole interaction in the spin-symmetric and antisymmetric channels (upper diagrams), and the particle-particle amplitude in the ${ }^{1} S_{1}$ channel (lower diagrams), shown at three different densities corresponding to $k_{F}=0.7,1.0,1.3 \mathrm{fm}^{-1}$. (The vertical dotted lines are drawn at $2 k_{F}$.) In the upper diagrams, the dashed lines represent the lowest-order phase-space averaged $G$-matrix elements, whereas the full lines denote the final solutions of the Babu-Brown equation. In the lower diagrams, the dashed lines show the ${ }^{1} S_{0}$ component of the bare $V_{14}$ potential, the full lines the contribution of the polarization (RPA) diagrams and the dotted lines the sum of the two contributions.

where $\boldsymbol{q}^{\prime}=\boldsymbol{k}_{1}-\boldsymbol{k}_{2}$. This equation is already a generalisation of the Babu-Brown equation, which considers particles on the Fermi surface only. We, in fact, assume as in Ref. [17] that this equation is valid outside the Fermi surface and that we may approximate the quantity $F$ (in the series of bubbles) by its value at $\boldsymbol{p}=\left(\boldsymbol{k}_{1}+\boldsymbol{k}_{2}\right) / 2$. Furthermore we assume (without writing it explicitly) that we may use in these bubbles the Landau angle expansion, limiting oursclves to the first two terms (see Ref. [17] for details). We now allow for a departure of $q=0$. Writing explicitly the $q$-dependence and the splitting in the symmetric and antisymmetric channels, we obtain

$$
\begin{aligned}
\left(\begin{array}{l}
F_{s}(q) \\
F_{a}(q)
\end{array}\right) & =\left(\begin{array}{l}
G_{s}(q) \\
G_{a}(q)
\end{array}\right) \\
& -\left(\begin{array}{cc}
\frac{1}{2} & \frac{3}{2} \\
\frac{1}{2} & -\frac{1}{2}
\end{array}\right)\left(\begin{array}{c}
\frac{F_{s}^{2}\left(q^{\prime}\right) \Pi\left(q^{\prime}\right)}{1-F_{s}\left(q^{\prime}\right) \Pi\left(q^{\prime}\right)} \\
\frac{F_{a}^{2}\left(q^{\prime}\right) \Pi\left(q^{\prime}\right)}{1-F_{a}\left(q^{\prime}\right) \Pi\left(q^{\prime}\right)}
\end{array}\right),
\end{aligned}
$$

where the summations on the intermediate states have been performed by assuming that the dependence upon $q^{\prime}$ could be replaced by taking an average value of $q^{\prime}$, Eq. (8d). Actually, this dependence is rather weak and $\left\langle q^{\prime}\right\rangle$, which is a function of $q$, is lying close to
$k_{F}$, as seen in Fig. 2. The solution of Eq. (14) using Eq. (10a) is given in the upper part of Fig. 3. We remind that the Landau parameters $F_{0}$ and $G_{0}$ for neutron matter are basically given by $F_{s}(0)$ and $F_{a}(0)$ They are shown in Fig. 4 and compared to other calculations $[14,18,19]$. One can see that the $q$-dependence of $F_{s}$ and $F_{a}$ is far from being negligible.

(iii) We construct the effective interaction $\Gamma$ in the particle-particle channel. The latter is obtained by summing the diagrams of Fig. 1(b) and is shown in the lower part of Fig. 3. It writes

$\Gamma_{S_{0}}\left(k, k^{\prime}\right)=\frac{1}{2 k k^{\prime}} \int_{\left|k-k^{\prime}\right|}^{k+k^{\prime}} d q q\left(\Gamma_{s}(q)-3 \Gamma_{a}(q)\right)$,

with $(i=s, a)$

$\Gamma_{i}(q)=V_{i}(q)+\frac{F_{i}^{2}(q) \Pi(q)}{1-F_{i}(q) \Pi(q)}$.

The Lindhard function $\Pi(q)$ was given in Eq. (10a). This is a particularly simple result, which holds for local interactions. That is why we constructed an equivalent local interaction. Antisymmetrization is guaranteed by the construction of the $F_{i}$ 's. 


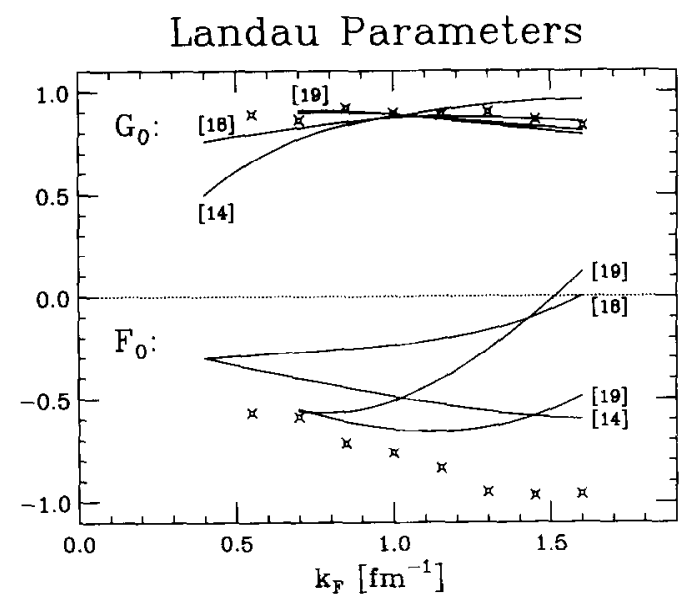

Fig. 4. The Landau parameters $F_{0}$ and $G_{0}$ in our model (crosses), in comparison with the results of Refs. $\left.\mid 14,18,19\right\}$ (full curves).

The density dependence of the pairing interaction is described in the lower part of Fig. 3. The same figure also shows that the $q$-dependence of the pairing interaction is only modified (compared to the bare interaction) for $q \lesssim 2 k_{F}$, which makes the correction rather long-ranged. This is due to the Lindhard function, which limits particle-hole excitations to long wavelengths. It can be seen that at low densities the polarization contribution is strongly repulsive, with a transition to attraction at higher densities. Here, however, the repulsion of the bare interaction dominates. Thus, the competing effects of density fluctuations ( attractive) and spin-density fluctuations (repulsive) in neutron matter appear to dominate in different regions of density in our model.

We solve numerically the gap equation, using Eqs. (15), (16) and the $V_{14}$ potential [20,21]. The results are given in Fig. 5. Comparing the values of the gap with and without the polarization part of the interaction, we can verify the effects expected from the discussion of the interaction above, namely, a strong reduction of the gap at low densities, and a slight enhancement at higher densities.

Often the following simple weak-coupling formula

$\Delta_{F} \approx 8 \frac{k_{F}^{2}}{2 m^{*}} \exp \left(\frac{-2}{N \Gamma_{S_{0}}\left(k_{F}, k_{F}\right)}\right)$,

is used to estimate the gap $[13,14]$. Roughly speaking, this formula amounts to assume that the pairing interaction acts in a limited range around $k_{F}$. We show in the same figure the gap predicted by this formula, magnified by a factor 10 . Obviously, the straightforward application of this approximate formula is completely misleading. This demonstrates the importance of the off-diagonal parts of the interaction away from the Fermi surface, the use of this approximation may be justified if pseudopotentials are introduced to represent the short range part of the interaction and especially its efffects on the off-diagonal part of the pairing interaction away from the Fermi surface. This is done in Ref. [14], on some phenomenological manner. We here preferred to solve the gap equation exactly, taking so account of the full interaction on a consistent manner.

We would finally like to compare our results to previous attempts of including medium polarization effects. In an early publication [13] the Landau parameters were used to estimate the effect of the polarization graphs, i.e., the momentum dependent effective particle-hole interactions $F_{s}(q), F_{a}(q)$ in Eq. (16) were replaced by Landau parameters for neutron matter, $F_{0}$ and $G_{0}$. Together with the use of the weakcoupling approximation, a strong reduction of the gap was predicted. In Ref. [7], neutron matter superfluidity was studied in the framework of second-order correlated-basis perturbation theory, corresponding to the inclusion of the first terms in the series of polarization diagrams, besides other diagrams of second order that we do not consider. A strong reduction of the gap by about a factor four was predicted in that work. Apart from the restriction to second order diagrams, which has been shown to underestimate the gap [14], rather 


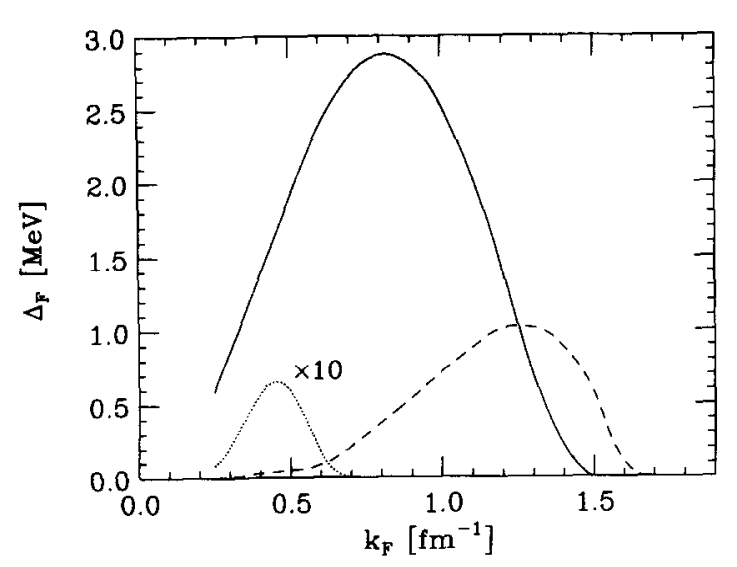

Fig. 5. The gap $\Delta_{F}$ in the ${ }^{1} S_{4}$ channel of neutron matter as a function of the Fermi momentum $k_{F}$ in various approximations. Full curve: using the direct interaction with the bare $V_{14}$ potential. Dotted curve: weak-coupling approximation for the same interaction, magnified by a factor 10. Dashed curve: including the polarization part of the interaction. The weak-coupling approximation in this case is zero.

strong approximations concerning the behaviour of the polarization part away from the Fermi surface were made, namely only its value on the Fermi surface was evaluated explicitly, and subsequently converted into a scaling factor for the lowest order, direct, interaction. This again amounts to neglecting the momentum dependence of the induced interaction and is a questionable procedure. Finally, our results can be compared with those of the polarization potential model of Ref. [14], which basically comprises the same set of diagrams for the induced interaction as in the present work. It does not enforce a local approximation, but relies on a somehow arbitrary cut-off of the repulsive part of the potential. The main result of that work is a repulsive effect of the induced interaction in the spinsymmetric channel, whereas the spin-antisymmetric one was basically unaffected, in agreement with our results. They finally used the weak-coupling formula (with a momentum domain of width equal to $k_{F} / 2$ ) for calculating the gap.

A numerical comparison between these works and ours, concerning the maximum value of the gap and the approximate domain where the gap is significantly different from zero, is provided by the table 1 .

We thus conclude that the addition of the set of polarization diagrams to the bare potential considerably reduces the gap to values of the order of $1 \mathrm{MeV}$ at the most. Within the frame of the particular choice of
Table 1

\begin{tabular}{lll} 
& $\left(\Delta_{F}\right)_{\max }(\mathrm{MeV})$ & Density $\left(\mathrm{fm}^{-3}\right)$ \\
\hline Ref. $[13]$ & 0.64 & $\sim 0.014$ \\
Ref. [7] & 0.5 & $0.004-0.017$ \\
Ref. [14] & $1.2-1.5$ & $0.007-0.066$ \\
This work & 0.9 & $0.034-0.114$ \\
\hline
\end{tabular}

graphs adopted here, our results should be considered as an upper limit. The averaging procedure used to solve Eq. (14) is presumably producing a too strongly attractive pairing effective interaction. Indeed, our predicted value of $F_{0}$ is more negative than the value of Ref. [14] (at large values of $k_{F}$ at least), which basically used the same interaction.

Such a small gap may have drastic consequences on the pinning mechanism of vortices in the neutron star crust. The pinning energy would be much lower than the usual estimates [22]. The strong pinning regime would be unprobable and pinning would occur in the inner crust only. Whether this would be sufficient to explain the observed glitches [23] in the neutron star rotation is still an open question.

This work was supported by the EU contract ERBCHRX-CT92-0075.

\section{References}

[1] D. Pines, J. de Phys. 41 (1984) C2 111

[2] J. Bardeen, L.N. Cooper and J.R. Schrieffer, Phys. Rev. 108 (1957) 1175.

[3] L.P. Gor'kov, Phys. Rev. C 29 (1984) 1207.

[4] A.B. Migdal, Theory of finite systems and applications to atomic nuclei (Benjamin, New York, 1964).

[5] P. Nozières, Le problème à $N$ corps (Dunod, Paris, 1963).

[6] P. Ring and P. Schuck, The nuclear many-body problem (Springer Verlag, Berlin, 1980).

[7] J.M.C. Chen, J.W. Clark, E. Krotschek and R.A. Smith, Nucl. Phys. A 451 (1986) 509; J.M.C. Chen, J.W. Clark, R.D. Davé and V.V. Khodel Nucl. Phys. A 555 (1993) 59.

181 M. Baldo, J. Cugnon, A. Lejeune and U. Lombardo, Nucl. Phys. A 515 (1990) 409

19] M. Baldo, J. Cugnon, A. Lejeune and U. Lombardo, Nucl. Phys. A 536 (1991) 349.

[10] S. Babu and G.E. Brown, Ann. Phys. (N.Y.) 78 (1973) 1.

[11] D. Pines, Proc. 12th Int. Conf. on low temperature physics, ed. by E. Kandu (Kligatu, Tokyo, 1971) p. 10.

[12] J.A. Niskanen and J.A. Sauls, unpublished, cited in Ref. |7|. 
113| J.W. Clark, C.-G. Källman, C.-H. Yang and D.A. Chakkalakal, Phys. Lett. B 61 (1976) 331

14| T.L. Ainsworth, J. Wambach and D. Pines, Phys. Lett. B 222 (1989) 173;

J. Wambach, T.L. Ainsworth and D. Pines, Nucl. Phys. A 555 (1993) 128

115 L. Amundsen and E. Ostgaard, Nucl. Phys. A 437 (1985) 487.

| $16 \mid$ O. Sjöberg, Ann. Phys. N.Y. 78 (1973) 39.

| $17 \mid$ S.-O. Bäckmann, G.E. Brown and J.A. Niskanen, Phys. Rep. 124 (1985) 1.

| $18 \mid$ S. -O. Bäckmann, C.-G. Källman and O. Sjöberg, Phys. Lett. B 43 (1973) 263
[19] A.D. Jackson, E. Krotschek, D.E. Meltzer and R.A. Smith, Nucl. Phys. A 386 (1982) 125.

[20] R.B. Wiringa, R.A. Smith and T.L. Ainsworth, Phys. Rev. C 29 (1984) 1207.

[21] I.E. Lagaris and V.R. Pandharipande, Nucl. Phys. A 359 (1981) 331.

[22] G. Lazzari and F.V. De Blasio, il Nuovo Cimento Vol. A 108 N.3 (1995) 313.

[23] M.A. Alpar, P.W. Anderson, D. Pines and J. Shaham, Astrophys. J. 249 (1981) L33. 